\title{
Evaluating the therapeutic effect of tumor treating fields (TTFields) by monitoring the impedance across TTFields electrode arrays
}

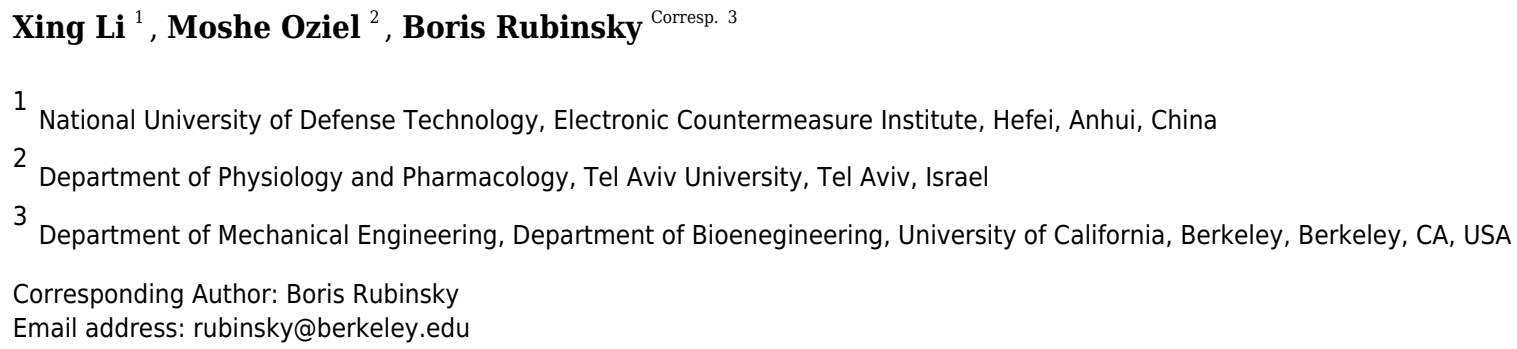

Background: Tumor Treating Fields (TTFields), is a novel, non-invasive tissue ablation technology for treatment of cancer. Tissue ablation is achieved through the continuous delivery of a narrow range of electromagnetic fields across a tumor, for a period of months. TTFields are designed to affect only cells that divide and to interfere with the cell division process. The therapy is monitored with MRI imaging, performed every couple of months. Current technology is unable to assess the treatment effectiveness in real time. Methods: We propose that the effect of the treatment can be assessed, in real time, by continuously measuring the change in electrical impedance across the TTFields delivery electrode arrays. An in vitro anatomic skull experimental study, with brain and tumor mimics phantom tissues was conducted to confirm the potential value of the proposed monitoring system. Results: Experiments show that measuring the change in the impedance amplitude between opposite TTFields electrode arrays, at a typical TTFields treatment frequency of $(200 \mathrm{kHz})$, can detect changes in the tumor radius with a sensitivity that increases with the radius of the tumor. The study shows that TTFields electrode arrays can be used to assess the effectiveness of TTFields treatment on changes in the tumor dimensions in real time, throughout the treatement. This monitoring system may become a valuable addition to the TTFields cancer treatment technology. It could provide the means to continuously assess the effectiveness of the treatment, and thereby optimize the design of the treatment protocol. 


\section{Evaluating the therapeutic effect of tumor treating fields (TTFields) by monitoring the impedance across TTFields electrode arrays}

\section{Xing Li $^{{ }^{*}}$, Moshe Oziel ${ }^{2}$, Boris Rubinsky ${ }^{3}$}

${ }^{1}$ Electronic Countermeasure Institute, National University of Defense Technology, Hefei, Anhui, China

${ }^{2}$ Department of Physiology and Pharmacology, Tel-Aviv University, Tel Aviv, Israel.

${ }^{3}$ Department of Mechanical Engineering, Department of Bioengineering, University of California Berkeley, Berkeley, CA, USA.

Corresponding Author:

Boris Rubinsky

6124 Etcheverry Hall UC Berkeley, Berkeley, CA 94720, USA

Email address: rubinsky@berkeley.edu

.

(1)

2

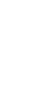

.

(1)

.

1
3

.

8


40 Abstract

41 Background: Tumor Treating Fields (TTFields), is a novel, non-invasive tissue ablation 42 technology for treatment of cancer. Tissue ablation is achieved through the continuous delivery of

43 a narrow range of electromagnetic fields across a tumor, for a period of months. TTFields are 44 designed to affect only cells that divide and to interfere with the cell division process. The therapy 45 is monitored with MRI imaging, performed every couple of months. Current technology is unable 46 to assess the treatment effectiveness in real time.

47 Methods: We propose that the effect of the treatment can be assessed, in real time, by continuously measuring the change in electrical impedance across the TTFields delivery electrode arrays. An in vitro anatomic skull experimental study, with brain and tumor mimics phantom tissues was conducted to confirm the potential value of the proposed monitoring system.

51 Results: Experiments show that measuring the change in the impedance amplitude between opposite TTFields electrode arrays, at a typical TTFields treatment frequency of $(200 \mathrm{kHz})$, can detect changes in the tumor radius with a sensitivity that increases with the radius of the tumor. The study shows that TTFields electrode arrays can be used to assess the effectiveness of TTFields treatment on changes in the tumor dimensions in real time, throughout the treatment. This monitoring system may become a valuable addition to the TTFields cancer treatment technology. It could provide the means to continuously assess the effectiveness of the treatment, and thereby optimize the design of the treatment protocol.

Keywords: Tumor Treating Fields, impedance detection, brain tumor, tumor condition,

\section{Introduction}

Tumor Treating Fields (TTFields) is a minimally invasive, non-contact, tissue ablation technology that employs intermediate-frequency $(100-300 \mathrm{kHz})$, and low-intensity $(<3 \mathrm{~V} / \mathrm{cm})$ electric fields to inhibit the growth of dividing (cancer) cells (Kirson et al., 2004). Clinical evidence shows that the delivery of TTFields prolongs the survival time of glioblastoma multiform (GBM) patients, without obvious side effects (Guberina et al., 2020), (Mun et al., 2018). The Food and Drug Administration (FDA) has approved the use of TTFields for GBMtreatment (Guberina et al., 2020), (Mun et al., 2018). In clinical practice, TTFields are delivered in paired orthogonal directions, left-right and anterior-posterior using insulated ceramic

74 the placement of the electrodes in relation to the tumor, is shown in Fig. 1A. The electrodes are

75 rigidly attached to the shaved scalp and are connected to a portable power generator. To

76 maximize the intensity of the electric fields delivered to the tumor, the placement of the 
77 treatment electrodes is individualized to each patient, using the patient own baseline magnetic

78 resonance image (MRI) of the brain to determine the optimal placement. NovoTal System

79 (NovoTal, USA) is a commercial software for optimization of the TTFields electrode

80 placements. A detailed description of the way in which the electrode placements and the choice

81 of treatment parameters are optimized is given in (Trusheim et al., 2017). TTFields treatment is

82 substantially different from other clinical tissue ablation treatments. In most conventional tissue

83 ablation treatments, such as microwave, radiofrequency, cryosurgery, focused ultrasound,

84 irreversible electroporation, radiation therapy, the surgical procedure is minutes long. The

85 ablative energy is delivered under real time medical imaging. The success of these treatments

86 can be assessed shortly after the end of the procedure, with conventional medical imaging.

87 Unlike other tissue ablation treatments, which affect all the cells in the treated volume, TTFields

88 affect only the tumor cells that divide. The TTFields electromagnetic fields interfere with the cell

89 division process and stop the cell division (Li, Yang \& Rubinsky, 2020). Therefore, TTFields

90 are delivered for long periods to affect cells that divide, whenever they are dividing. In

91 conventional TTFields treatment, the electric fields are delivered for many months and even

92 years (Davies, Weinberg \& Palti, 2013). The patient activates a portable power supply to deliver

93 the treatment. The treatment usually lasts for up to 18 hours per day (Mrugala et al., 2014). The

94 effect of TTFields is frequency depended and the optimal frequency can vary with cell type

95 (Giladi et al., 2015). For GBM, the optimal frequency is thought to be about $200 \mathrm{kHz}$. For

96 technical and economic reasons the success of the treatment on GBM is assessed by a limited

97 number of follow-up MRI scans, which are usually performed every two months (Trusheim et

98 al., 2017), (Hottinger, Pacheco \& Stupp, 2016). If these once every two months MRI scans show

99 that the treatment is not successful, the remedy is to change the treatment parameters. However,

100 GBM is an aggressive disease in which the tumor can grow fast. A technology for real time

101 monitoring of the tumor growth or recession during the actual TTFields treatment could provide

102 timely feedback to the physician on the efficacy of the treatment parameters.

104 Typical TTFields treatment protocols are as follows. After clinical verification of the nature of 105 the tumor, MRI is used to precisely determine the location of the tumor and its dimensions.

106 Then, the MRI images are used with an optimization algorithm, the NovoTal System (Trusheim 107 et al., 2017) (NovoTal, USA) to calculate the optimal placement of the treatment electrodes, in 
108 such a way that the resulting electromagnetic fields are focused onto the tumors, throughout the 109 months long application of the treatment. In this paper we show that the way in which TTFields 110 are delivered across tumors, with electrodes fixed to the skull at a location calculated to deliver 111 the electric fields precisely to the tumors, makes the treatment electrodes particularly suitable for 112 real time monitoring of tumor size through the measurement of the electrical impedance between 113 the electrodes. Changes in the tumor size will affect the electrical impedance between electrodes 114 because normal and malignant brain tissues have different electric properties (Latikka \& Eskola, 115 2019).

117 The head can be viewed as a complex electric circuit network, represented by a black box. The 118 electrode arrays on the scalp are the accessible nodes to the black box network, which is the 119 head, as shown in a schematic way in Fig. 1B. Because of the difference in electrical properties 120 between the normal and malignant brain tissue (Latikka \& Eskola, 2019), any changes in the 121 tumor size and composition will lead to changes in the impedance measured across the black box 122 circuit network that is the head. Therefore, we suggest that monitoring changes in the electrical 123 impedance between the TTFields delivery electrodes could be used to detect changes in the 124 tumor treated by these electric fields, throughout the treatment, in real time. Once changes are 125 detected, conventional medical imaging can be used to investigate the nature of these changes. 126 This could replace randomly chosen times for medical imaging follow-up with follow ups that 127 are clinically significant. Furthermore, if no changes in the impedance between the TTFields 128 electrodes are detected, it may be an indication that the treatment is ineffective and that the treatment parameters should be modified. It should be emphasized, that measuring changes in electrical impedance between electrodes to monitor changes in the composition of tissue between

131 the electrodes is not new. In fact, it serves as the basis for imaging techniques known as “electrical impedance tomography” (EIT) (Escobar, 2020) and magnetic induction tomography

133 (MIT) (Griffiths, 2001). A closely related use of the concept described in this paper is to 134 clinically monitor internal bleeding in the brain (Gonzalez et al., 2013), and to monitor cerebrovascular autoregulation (Oziel et al., 2016).

137 This paper is a first order feasibility experimental study, whose goal is to asses if measuring changes in electrical impedance between TTFields electrodes is sensitive enough to detect 
139 changes in the tumor size. In this study we explore the feasibility of the concept with a first order

140 experimental model that employs a geometrically accurate head filled with a gel with electrical

141 properties of brain tissue and in which the tumor is simulated with a tuber, with electrical

142 properties of a tumor. The TTFields electrodes are attached to the skull and the experiments

143 measure the changes in electric impedance between the TTFields electrodes as a function of the

144 simulated tumor size and location. The other anatomic details of the head are considered fixed

145 throughout the treatment and represented by the black box circuit network in Figure 1B. This

146 model is a first order approximation of human and tumor anatomy and is used to evaluate the

147 feasibility of the concept.

149 We should also note that GBMS are not simply solitary masses but rather are often deeply 150 infiltrating tumors, with scattered neoplastic cells extending out far from any observable mass on 151 MRI. Moreover, the brain has ventricles which can take up a substantial volume of the cranial 152 vault. However, the intent of the technique introduced in this study is to evaluate the feasibility 153 of detecting changes in the main tumor mass from TTFields treatment by monitoring changes in 154 impedance between the TTFields electrodes at a given frequency and for a fixed location of the 155 TTField electrodes relative to the tumor. It should be emphasized that conventional monitoring 156 of the effect of TTFields treatment is also done by monitoring the size of the tumor with MRI. 157 The clinical efficiency of the treatment with TTFields and MRI means of treatment monitoring, 158 has been demonstrated in thousands of treatment, worldwide.

While the brain and the tumor have a complex anatomy and composition, our measurements are geared to compare only the changes in impedance across the brain, before and after the TTFields treatment, with an eye towards the changes in the mass of the main tumor, as MRI does. The concept of using electromagnetic properties "changes" across the brain, to detect physiological 165 studies, e.g. (Oziel et al., 2016), (Kellner et al., 2018), (Venkatasubba Rao et al., 2018). 
170 of a durable polymer (Anatomy Warehouse, USA). The overall size is about $170 \mathrm{~mm} \times 145 \mathrm{~mm}$.

171 The TTFields electrodes were placed on the skull in a way that precisely simulates clinical

172 practice. Electrode arrays were attached to the skull at four locations, anterior, posterior, left and

173 right, as shown in Fig.1C. At each location there are 6 electrodes shorted together and connected

174 to one measurement output. The $10 \mathrm{~mm}$ radius electrodes were designed to be similar to

175 TTFields treatment electrodes (Korshoej et al., 2017). The TTFields simulating electrode arrays

176 are made of a copper tape (Bertech, USA) with an insulated adhesive layer with which the

177 electrodes are attached to the surface of the skull. The dimensions and placement of the

178 electrodes on the skull and the output electric wire from each array are shown in Fig. $1 \mathrm{C}$. The

179 brain was simulated by a gel with electrical properties similar to those of bulk brain tissue

180 (Pomfret \& et al, 2013) (Kandadai M A, Raymond J L, 2012). The gel was made of $4 \%$ alginate

181 sodium $+2 \% \mathrm{NaCl}+94 \%$ deionized water. We have verified that this composition has electric

182 properties similar to those of the brain in the frequency range of interest (test is not shown here).

183 The GBM brain tumor, has a higher water content and an electrical conductivity that is several to

184 ten times higher than the surrounding healthy tissue normal brain tissue (Foster \& Schwan,

185 1989), which can provide sufficient sensitivity to evaluate the tumor change via impedance shift. The

186 potato is used as a substitute for the tumor because the potato electrical conductivity at the

187 frequency range of interest (Gratz et al., 2021) is similar to that of a tumor (Latikka \& Eskola,

188 2019).

189

190 The potato was shaped into a sphere and placed at a predetermined location in the skull. Then,

$191800 \mathrm{ml}$ gel was poured into the inverted skull, around the potato, as shown in Fig 2B. The

192 coordinates used for the placement of the potato (tumor) are shown in a schematic in Fig. 2C.

193 The electrode arrays were connected to a precision Impedance Analyzer (Agilent 4294A, USA)

194 (Fig. 2A) to measure the impedance between each two opposite electrode arrays. Various

195 dimensions of the potato sphere and their placement at various locations, were used to study the

196 effects of tumor size and location on the impedance between the TTFields electrode arrays.

197 Typically, TTFields are delivered in the frequency range of from $100 \mathrm{kHz}$ to $300 \mathrm{kHz}$ (Fabian et

198 al., 2019), at which they produce the maximal therapeutic effect. 
200 In this study, the impedance measurements were made at a frequency of $200 \mathrm{kHz}$, which is the

201 preferred frequency used for clinical TTFields treatments. We report here only results from the

202 change in amplitude measurements. We have shown in a series of previous studies that phase

203 shift measurements at frequencies lower than $0.1 \mathrm{MHz}$ cannot detect changes in tissue

204 e.g.(Gonzalez \& Rubinsky, 2006; González \& Rubinsky, 2006; Gonzalez, Horowitz \&

205 Rubinsky, 2007) (González et al., 2009). Therefore, we report only experimentally measured

206 changes in the impedance amplitude that resulted from the insertion of various tumor emulators

207 in the skull, i.e. $\Delta \mathrm{Z}=\left|\mathrm{Z}_{2}\right|-\left|\mathrm{Z}_{1}\right|$.

208

\section{Results}

In clinical practice, the TTFields electrode arrays are placed rigidly on the scalp at locations predetermined by the NovoTal System (NovoTal, USA), in such a way as to optimize the optimal delivery of the electric fields to the treated tumor. The treatment electrode arrays are usually placed in two opposite configurations, orthogonal to each other, which is considered to be the optimal layout for delivering the TTFields dose to the tumor (Korshoej et al., 2017). Therefore, the impedance between TTFields electrode arrays can be measured in two configurations, opposite and adjacent. Previous studies on placement of electrical impedance tomography (EIT) electrodes have shown that opposite electrodes measurements are more sensitive than adjacent electrode measurements (Koksal \& Eyuboglu, 1995). Therefore, our experiments were carried out with measurements made between opposite electrode arrays.

To establish a base line, the impedance between electrodes with the brain tissue phantom (gel) only, i.e. without the tumor, was measured first. At a frequency of $200 \mathrm{kHz}$, the values of the impedance between the electrode arrays on the anatomical skull, filled only with the gel, without the simulated tumors, along the $\mathrm{x}$-axis and the $\mathrm{y}$-axis, are $372.25 \Omega$ and $606.77 \Omega$, respectively, this measurement was followed by experiments in which we inserted the tumor potato model at eight different locations on the $\mathrm{x}$-axis and $\mathrm{y}$-axis, in the simulating brain tissue gel. In these experiments the impedance was measured between the two opposite TTFields electrode arrays, at $200 \mathrm{kHz}$. Four repeated measurements were made for each experimental case. Fig. 3A gives the experimental results showing the effect of tumor size and location on the changes in impedance relative to the baseline measurements. The maximal positive and negative deviations relative to the mean value were calculated from four repeat and are marked as error bars on the data points. It is evident that the change in impedance increases with the radius of the tumor. The figures show that it is possible to distinguish between the data point for every $5 \mathrm{~mm}$ increase in consecutive tumor radiuses, with a statistical significant difference of $\mathrm{F}=39.81>\mathrm{F} \_$crit $=3.49$ and $\mathrm{P}=1.63 \times 10^{-6}<0.05$, analyzed by ANOVA in Excel. 


\section{Discussion}

240 The experimental results demonstrate that changes in tumor size can be monitored with typical TTFields electrodes and used to provide continuous information on the effects of the treatment on the tumor dimensions, with a resolution of at least $5 \mathrm{~mm}$. The experimental data can be also used used to assess the sensitivity of the experimental impedance change measurements to the initial tumor radius. The first order derivative is a local measure of how changes in the abscissa affect changes in the ordinate. We performed this sensitivity analysis for two extreme conditions. Case A which deals with tumors at $\mathrm{x}=0$ which is the furthest location from the electrodes. Case B deals with tumors on $y=40$, which is the proximate location to the electrodes. To calculate the derivative continuously, as a function of initial tumor radius, we used a cubic interpolation of the experimental points. The plots of the derivatives as a function of initial tumor radius are shown Fig. 3B, for cases A and B. It is evident that the sensitivity (first order derivative) increases with an increase in the initial radius of the phantom tumors in an exponential way and is larger for tumors that are close to the electrodes. This study shows that this technology is more effective at assessing the success of the TTFields treatment on larger tumors. This has clinical value, because the larger the tumor the larger the peril to the patient and the less time there is to optimize the treatment parameters.

In any real measurement system, the measurement noise is unavoidable. This section will discuss the effect of measurement noise on the accuracy. The conventional definition, of the Signal to

Where, $Z_{\mathrm{s}}$ is the accurate measured impedance, $Z_{\mathrm{n}}$ is the measurement impedance noise.

$$
Z_{\mathrm{n}}=Z_{\mathrm{s}} \cdot 10^{-\mathrm{SNR} / 20}(2 \cdot \text { rand }-1)
$$

Where, the rand is a function that generates a random number between $0-1$. 
As discussed earlier the baseline value of the measured impedance in the absence of tumors is between $372 \Omega$ and $605 \Omega$. We perform here a calculation for a roughly median impedance measurement of $500 \Omega$. The highest resolutions of the impedance change for different noise levels was calculated from Eq. (4) and results show that, as expected, the resolution is improved with an increase in SNR. When the SNR is from $40 \mathrm{~dB}$ to $60 \mathrm{~dB}$, the resolution of impedance changes with the tumor size between $5 \Omega / \mathrm{mm}$ and $0.5 \Omega / \mathrm{mm}$. This implies that changes in tumor radius of $1 \mathrm{~mm}$ can be reliable detected only if it is yields a larger than $0.5 \Omega$ change in impedance. Our experimental data analysis in Fig. 3B, shows that this noise level is too high to detect $1 \mathrm{~mm}$ changes in tumor radius, even for a tumor as large as $20 \mathrm{~mm}$. At $20 \mathrm{~mm}$ initial tumor radius, the first derivative is less than or close to $5 \Omega / \mathrm{mm}$. However a SNR of $50 \mathrm{~dB}$ and higher is quite acceptable, in particular for larger tumors. Figure 3B shows that the first derivative for a tumor larger than $10 \mathrm{~mm}$ is about $1.21 \Omega / \mathrm{mm}(\mathrm{y}=40 \mathrm{~mm}$, black curve in Fig. 3B) and $0.51 \Omega / \mathrm{mm}(\mathrm{x}=0$, red curve in Fig. 3B). This result suggests that in practice, the SNR of the impedance measurement system should be higher than $50 \mathrm{~dB}$. This is a reasonable level. The accuracy of the commercial Impedance Analyzer Agilent 4294A that was used in the experimental part of this study is $62 \mathrm{~dB}( \pm 0.08 \%$, data from the instrument manual). Higher precision electronics can be used to ensure an even higher SNR, for example precision electric bridges.

As for the simulated skull model, obviously, it is not an exact replica of the real human head and the real GBM tumor. In reality, the brain tissue is inhomogeneous with ventricles and the GBM tumor can have extensions in the brain. However, the materials used in the experiments have similar electric properties as the brain and the tumor. Therefore, considering the head a black box with constant electric properties, except for the change in size and location of the simulated tumor, can provide a qualitative assessment in a first order model examination of the effect of tumor changes on impedance across TTFields electrodes.

\section{Conclusion}

TTFields are a relative new tissue ablation technology that treats cancer by affecting the division process in cancer cells. Treatment is done over periods of months and years and there is no simple technology to monitor the effectiveness of the treatment over time. Here we examined the idea that measuring the change in impedance across the electrode arrays that deliver the TTFields treatment could be used to monitor in real time the temporal changes in the tumor size. An in vitro skull experimental study has confirmed the potential value of the proposed monitoring system. Preliminary data suggests that measuring the change in impedance amplitude between opposite TTFields electrode arrays, at the frequency of the typical TTFields treatment 
316

317

318

319

320

321

322

323

324

325

326

327

328

329

330

331

332

333

334

335

336

337

338

339

340

341

342

343

344

345

346

347

348

349

350

parameters, can detect changes in the tumor radius with a resolution that increases with an increase in the initial radius of the tumor. This technique can be easily implemented by adding an impedance measurement function to the original commercial TTFields treatment device. The electrode arrays on the scalp can be designed to serve as a means to deliver the TTFields and as a sensor to measure the electrical impedance, between them. Obviously this is a first stage feasibility study that needs to be verified with clinical studies. If successful, this monitoring system may be a valuable addition to the TTFields cancer treatment technology. It is possible that in future implementations of this technique, an MRI image based exact simulation of the brain and the tumor, such as that generated by the NovoTal System (Trusheim et al., 2017) (NovoTal, USA) could be used to fine tune the interpretation of the measurements of the change in impedance between the TTFields electrodes.

While the brain and the tumor have a complex anatomy and composition, our measurements are geared to compare only the changes in impedance across the brain, before and after the TTFields treatment, with an eye towards the changes in the mass of the main tumor, as MRI does. It should be emphasized that the technique described in this study is designed for monitoring the effect of the TTFields procedure on the tumor, if it grows or recedes. The technique is not designed as a means to detect tumors. It is used only after the tumors are detected with other techniques that are much more precise, such as MRI. There is no doubt that MRI is much more sensitive and provides much more information than the technique in this paper. The advantage of this technique over MRI is that it is much less expensive, can be done at the home of the patient and continuously monitors the success of the treatment. However, we anticipate that this technique will be used primarily as a means to alert the physician that an MRI is immediately needed because the tumor seems to keep growing despite the treatment and that perhaps changes in treatment parameters are needed. Currently, the MRI's are scheduled at the physician chosen time intervals without any relation to the success of the treatment.

\section{LIST OF FIGURES AND TABLE}

Fig. 1 TTFields treatment of GBM: (A) schematic of the configuration of electrode array on the skull, (B) equivalent lump model circuit, C) Photographs of the anatomical skull and the TTFields electrode arrays.

Fig. 2 The setup of electrical impedance detection experiment on in vitro skull model, (A) skull model, (B) simulated brain tissue (gel) and tumor (potato), (C) the coordinate system of the model. 
352 Fig. 3 A) Upper left row: schematics of locations of the tumor phantom. Lower left row:

353 experimentally determined impedance change relative to the brain with no tumor, as a function of

354 tumor size at different tumor locations on the $\mathrm{x}$ axis and $\mathrm{y}$ axis, error bars are shown for each data

355 point. B): The sensitivity to tumor changes. The first order derivative of the results in Fig $3 \mathrm{~A}$.

Tab.1 Highest resolutions for different noise levels

\section{References}

Davies AM, Weinberg U, Palti Y. 2013. Tumor treating fields: a new frontier in cancer therapy,. Ann. N. Y. Acad. Sci. 1291:86-95. DOI: Http://dx.doi.org/10.1111/nyas.12112.

Escobar RF. 2020. Electrical impedance tomography: hardware fundamentals and medical applications. Ingeniería Solidaria 16:https://doi.org/10.16925/2357-6014.2020.03.02. DOI: , http://dx.doi.org/ 10.16925/2357-6014.2020.03.02.

Fabian D, Eibl M del PGP, Alnahhas I, Sebastian N, Giglio P, Puduvalli V, Gonzalez J, Palmer JD. 2019. Treatment of glioblastoma (GBM) with the addition of tumor-treating fields (TTF): A review. Cancers 11:https://doi.org/10.3390/cancers11020174. DOI: 10.3390/cancers 11020174 .

Foster KR, Schwan HP. 1989. Dielectric properties of tissues and biological materials: a critical review. Critical reviews in biomedical engineering 17:25-104.

Giladi M, Schneiderman R, Voloshin T, Al E. 2015. Mitotic spindle disruption by alternating electric fields leads to improper chromosome segregation and mitotic catastrophe in cancer cells. Sci. Rep. 5:18046. DOI: http://dx.doi.org/ 10.1038/srep18046.

Gonzalez CA, Horowitz L, Rubinsky B. 2007. In vivo inductive phase shift measurements to detect intraperitoneal fluid. IEEE Transactions on Biomedical Engineering 54:953-956. DOI: 10.1109/TBME.2006.889183.

Gonzalez CA, Rubinsky B. 2006. The detection of brain oedema with frequency-dependent phase shift electromagnetic induction. Physiological Measurement 27:539-552.

González CA, Rubinsky B. 2006. A theoretical study on magnetic induction frequency dependence of phase shift in oedema and haematoma. Physiological Measurement 27:829838. DOI: $10.1088 / 0967-3334 / 27 / 9 / 006$.

Gonzalez CA, Valencia JA, Mora A, Gonzalez F, Velasco B, Porras MA, Salgado J, Polo SM, Hevia-Montiel N, Cordero S, Rubinsky B. 2013. Volumetric Electromagnetic Phase-Shift Spectroscopy of Brain Edema and Hematoma. PLoS ONE 8:e63223. DOI: 10.1371/journal.pone.0063223.

González C a, Villanueva C, Vera C, Flores O, Reyes RD, Rubinsky B. 2009. The detection of brain ischaemia in rats by inductive phase shift spectroscopy. Physiological measurement 30:809-19. DOI: 10.1088/0967-3334/30/8/006.

Gratz M, Schttroff F, Gall L, Zejma B, Simon F, Jaeger H. 2021. Advantages of ohmic cooking in the kilohertz-range - part I: Impact of conductivity and frequency on the heating uniformity of potatoes. Innovative Food Science \& Emerging Technologies 67:102595. DOI: https://doi.org/10.1016/j.ifset.2020.102595. 
395

396

397

398

399

400

401

402

403

404

405

406

407

408

409

410

411

412

413

414

415

416

417

418

419

420

421

422

423

424

425

426

427

428

429

430

431

432

433

434

435

436

437

438

439

440
Griffiths H. 2001. Magnetic induction tomography. Measurement Science and Technology 12:1126-1131. DOI: 10.1088/0957-0233/12/8/319.

Guberina N, Pöttgen C, Kebir S, Lazaridis L, Scharmberg C, Lübcke W, Lüdemann L. 2020. Combined radiotherapy and concurrent tumor treating fields (TTFields) for glioblastoma: Dosimetric consequences on non-coplanar IMRT as initial results from a phase I trial. Radiat. Oncol. 15:https://doi.org/10.1186/s13014-020-01521-7.

Hottinger AF, Pacheco P, Stupp R. 2016. Tumor treating fields: a novel treatment modality and its use in brain tumors. Neuro-oncology, 18:1338-1349. DOI: http://dx.doi.org/10.1093/neuonc/now182.

Kandadai M A , Raymond J L SGJ. 2012. Comparison of electrical conductivities of various brain phantom gels: Developing a "Brain Gel Model." Mater Sci Eng C Mater Biol Appl. 32:2664-2667.

Kellner CP, Sauvageau E, Snyder KV, Fargen KM, Arthur AS, Turner R, Alexandrov AV. 2018. The VITAL study and overall pooled analysis with the VIPS non-invasive stroke detection device. Journal of NeuroInterventional Surgery 10:1079-1084.

Kirson ED, Gurvich Z, Schneiderman R, Dekel E, Itzhaki A, Wasserman Y, Schatzberger R, Palti Y. 2004. Disruption of Cancer Cell Replication by Alternating Electric Fields. Cancer Research 64:3288-3295. DOI: 10.1158/0008-5472.CAN-04-0083.

Koksal A, Eyuboglu B. 1995. Determination of optimum injected current patterns in electrical impedance tomography. Physiol. Measurements 16:A 99. DOI: http://dx.doi.org/10.1088/0967-3334/16/3A/010.

Korshoej AR, Hansen FL, Thielscher A, Von Oettingen GB, Sørensen JCH. 2017. Impact of tumor position, conductivity distribution and tissue homogeneity on the distribution of tumor treating fields in a human brain: A computer modeling study,. PLOS ONE 12:e0179214. DOI: http://dx.doi.org/10.1371/journal.pone.0179214.

Latikka J, Eskola H. 2019. The resistivity of human brain tumours in vivo. Ann. Biomed. Rev. 47:706-713. DOI: http://dx.doi.org/10.1007/s10439-018-02189-7.

Li X, Yang F, Rubinsky B. 2020. A theoretical study on the biophysical mechanisms by which tumor treating fields affect tumor cells during mitosis,. IEEE Trans. Biomed. Eng., 67:2594-2602. DOI: Http://dx.doi.org/10.1109/TBME.2020.2965883.

Mrugala MM, Engelhard HH, Tran DD, et al. 2014. Clinical practice experience with NovoTTF$100 \mathrm{~A}^{\mathrm{TM}}$ system for glioblastoma: the patient registry dataset (PRiDe),. Semin. Oncol. 41:S4-S13. DOI: Http://dx.doi.org/10.1053/j.seminoncol.2014.09.010.

Mun EJ, Babiker H, Weinberg U, Craft et al. CM. 2018. Tumor-treating fields: a fourth modality in cancer treatment. Clin. Cancer Res., 24:266-275.

Oziel M, Hjouj M, Gonzalez CA, Lavee J, Rubinsky B. 2016. Non-ionizing radiofrequency electromagnetic waves traversing the head can be used to detect cerebrovascular autoregulation responses (vol 6, 21667, 2016). Scientific Reports 6:23875.

Pomfret R, et al. 2013. Investigation of the electrical properties of agarose gel: characterization of concentration using nyquist plot phase angle and the implications of a more comprehensive in vitro model of the brain,. Ann. Neurosci. 20:99-107. DOI: http://dx.doi.org/10.5214/ans.0972.7531.200305.

Trusheim J, Dunbar E, Battiste J, et al. 2017. A state-of-the-art review and guidelines for tumor treating fields treatment planning and patient follow-up in glioblastoma,. CNS Oncol 6:2943. DOI: Http://dx.doi.org/10.2217/cns-2016-0032.

Venkatasubba Rao CP, Bershad EM, Calvillo E, Al. E. 2018. Monitoring of Intracranial Fluid 
441 Shifts During Dialysis Using Volumetric Integral Phase-Shift Spectroscopy (VIPS): A 442 Proof-of-Concept Study. Neurocrit Care 28:117-126.

443 Welvaert M, Rosseel Y. 2013. On the definition of signal-to-noise ratio and contrast-to-noise

444

445

446

447 


\section{Figure 1}

Schematics and photographs TTFields treatment of TTFields electrode arrays.

Fig. 1 TTFields treatment of GBM: (A) schematic of the configuration of electrode array on the skull, (B) equivalent lump model circuit, C) Photographs of the anatomical skull and the TTFields electrode arrays. 

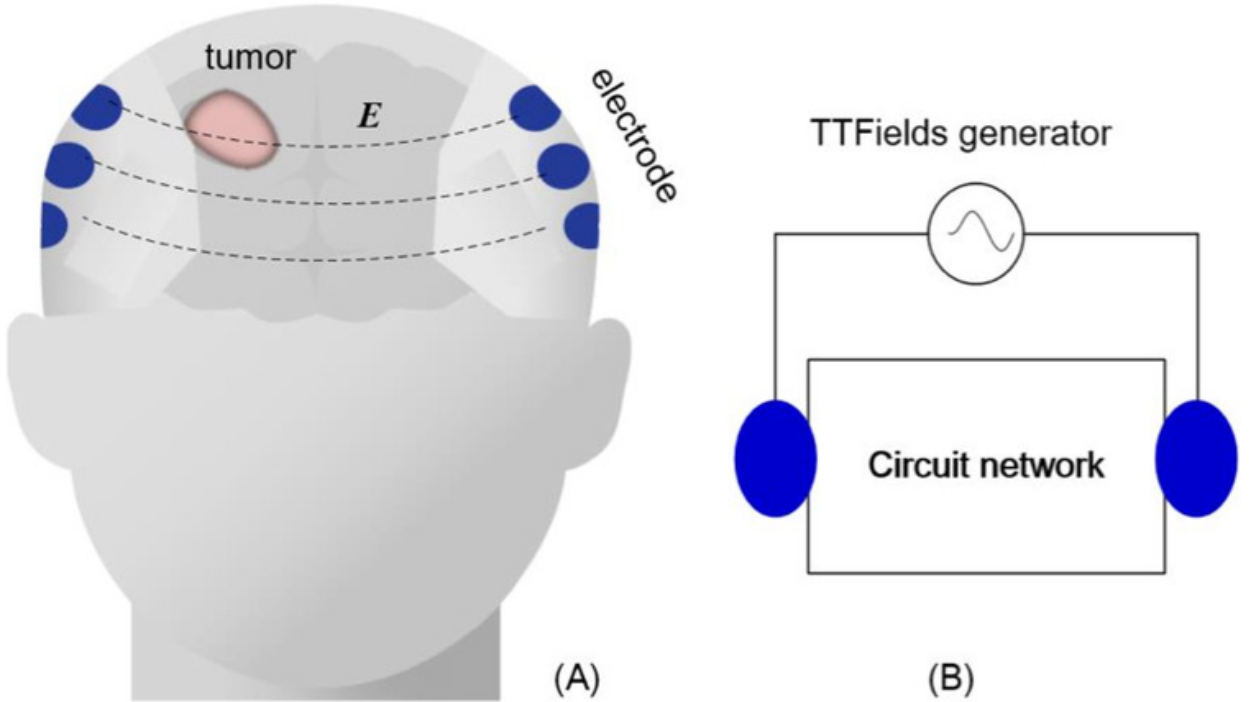

(A)

(B)
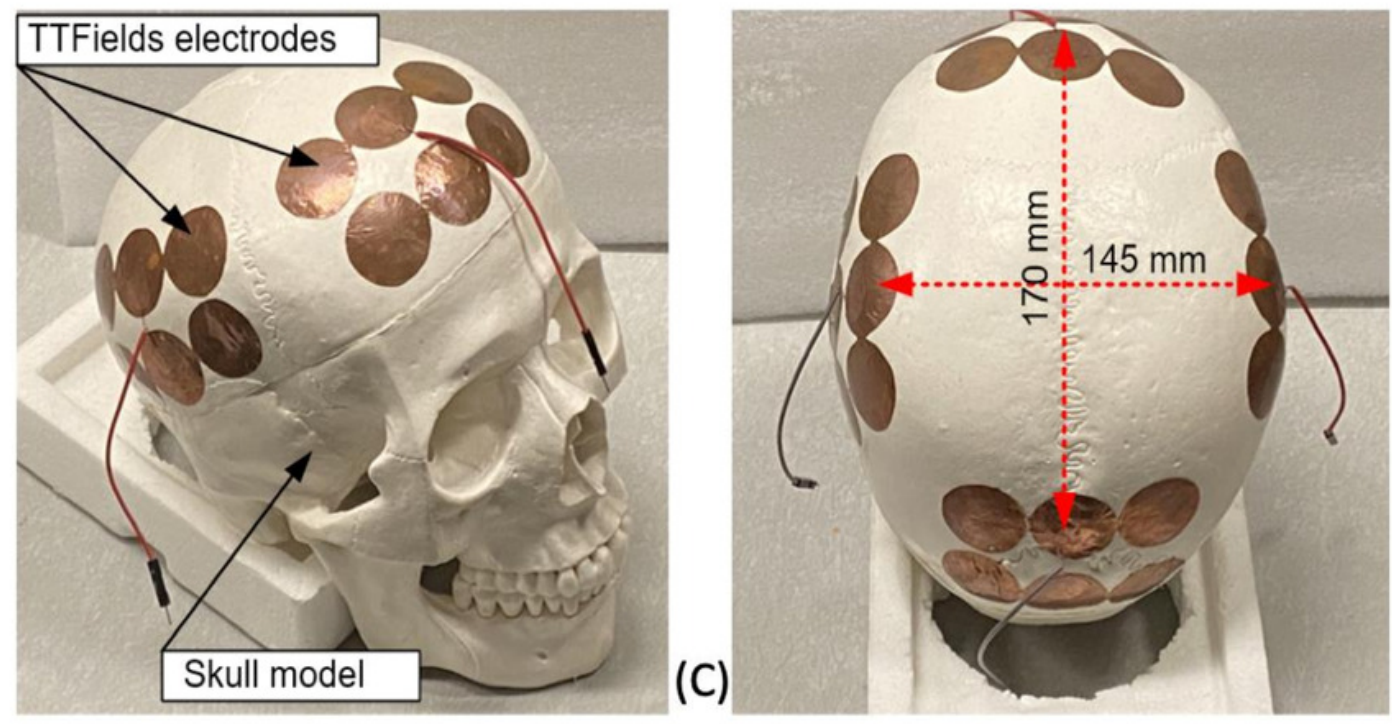

Fig. 1 TTFields treatment of GBM: (A) schematic of the configuration of electrode array on the skull, (B) equivalent lump model circuit, C) Photographs of the anatomical skull and the TTFields electrode arrays. 
Figure 2

Setup of experimental skull

Figure 2. The setup of electrical impedance detection experiment on in vitro skull model, $(A)$ skull model, (B) simulated brain tissue (gel) and tumor (potato), (C) the coordinate system of the model. 


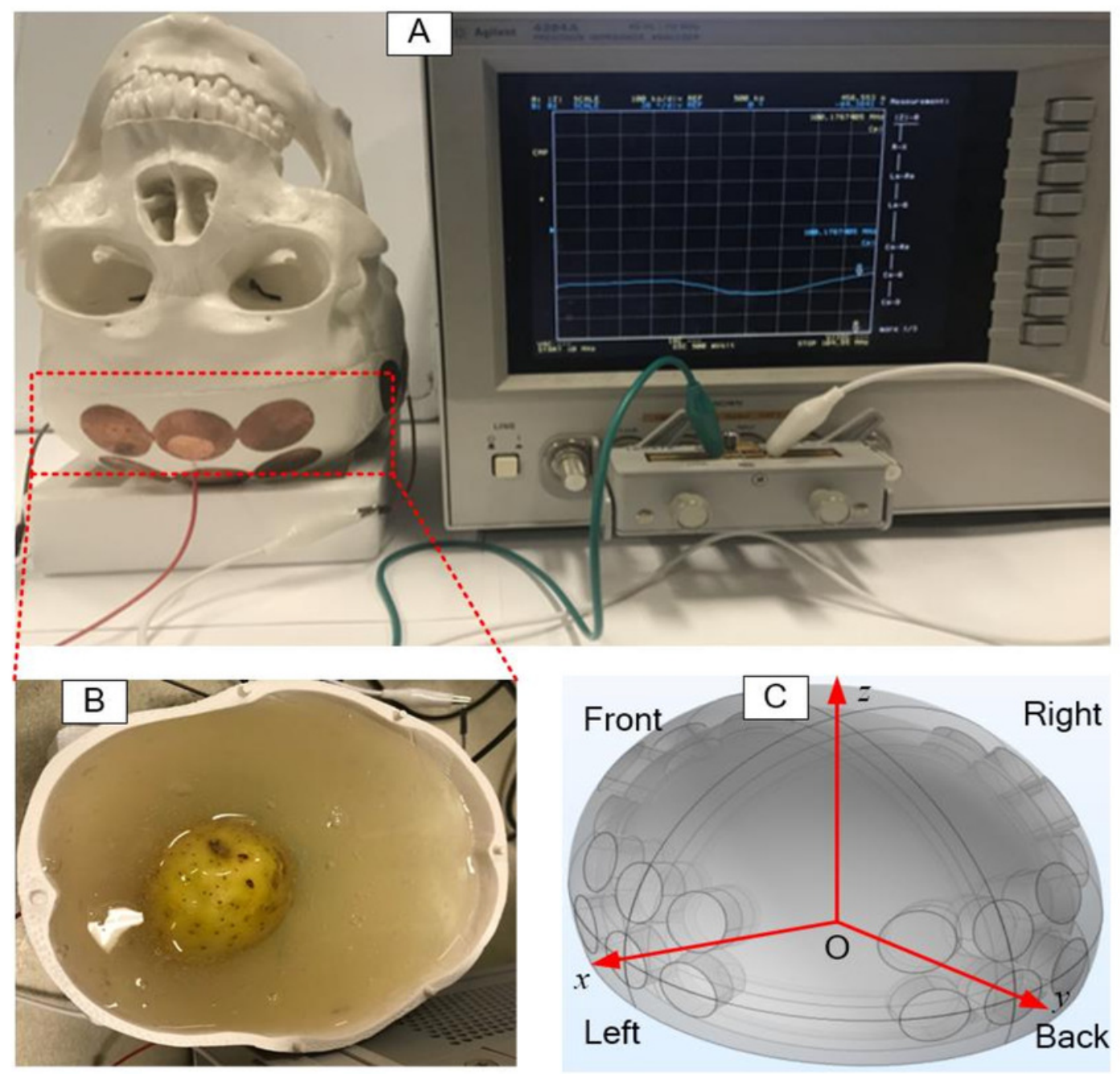

Fig. 2 The setup of electrical impedance detection experiment on in vitro skull model, (A) skull model, (B) simulated brain tissue (gel) and tumor (potato), (C) the coordinate system of the model. 


\section{Figure 3}

Impedance as a function of tumor radius

A) Upper left row: schematics of locations of the tumor phantom. Lower left row:

experimentally determined impedance change relative to the brain with no tumor, as a function of tumor size at different tumor locations on the $x$ axis and $y$ axis, error bars are shown for each data point. B): The sensitivity to tumor changes. The first order derivative of the results in Fig $3 \mathrm{~A}$. 
A
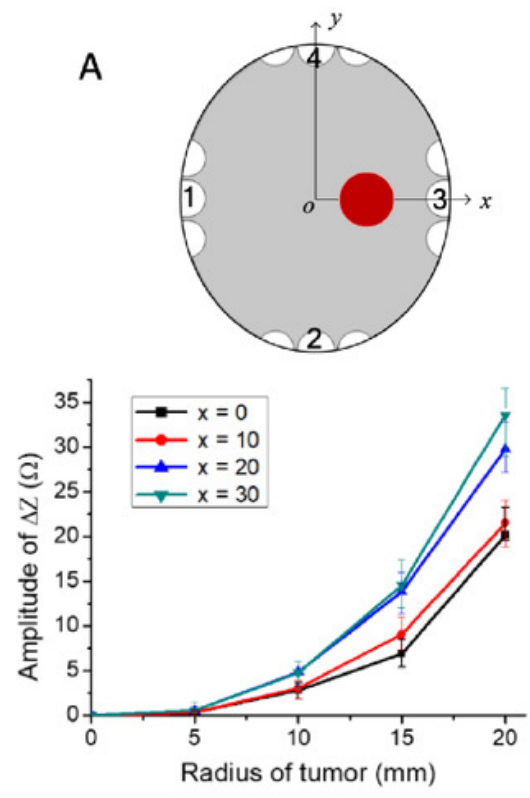

B
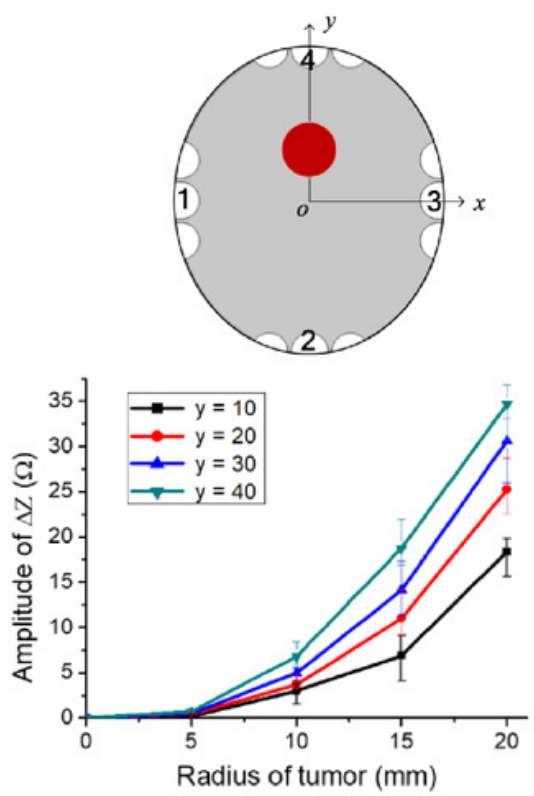

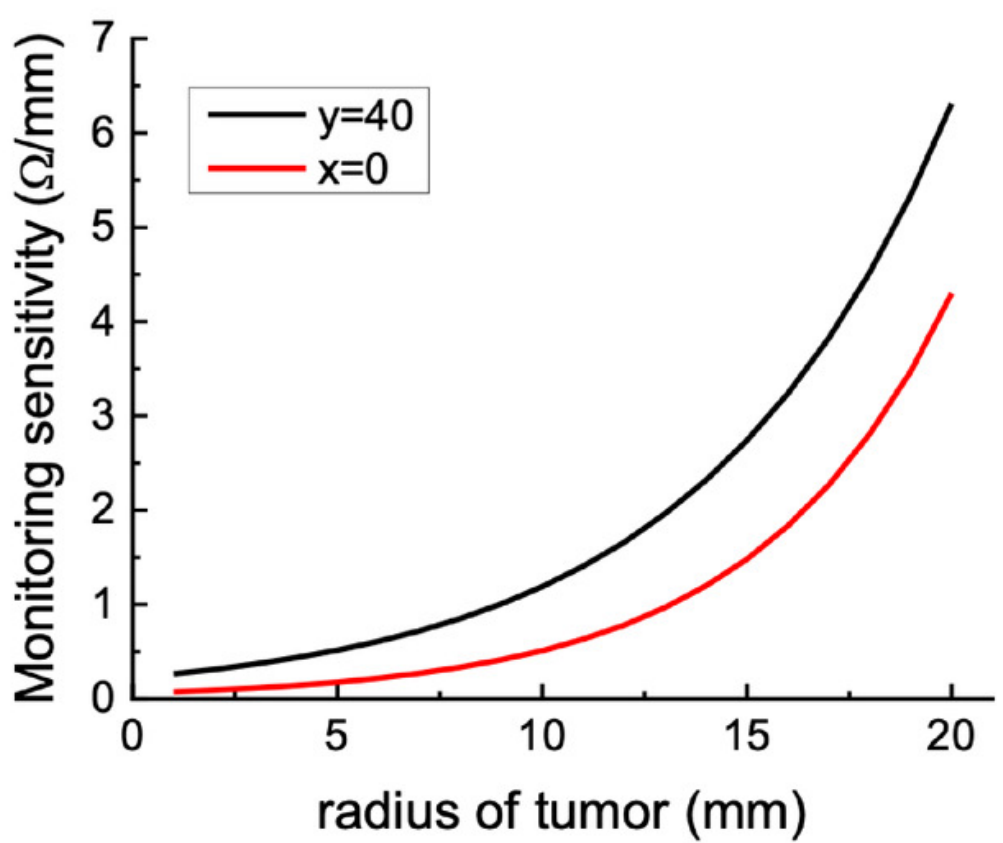

Fig. 3 A) Upper left row: schematics of locations of the tumor phantom. Lower left row: experimentally determined impedance change relative to the brain with no tumor, as a function of tumor size at different tumor locations on the $\mathrm{x}$ axis and $\mathrm{y}$ axis, error bars are shown for each data point. B): The sensitivity to tumor changes. The first order derivative of the results in Fig 3A. 
Table $\mathbf{1}$ (on next page)

Resolution as a function of noise level

Highest resolutions for different noise levels 


\begin{tabular}{llll}
\hline SNR $(\mathrm{dB})$ & 40 & 50 & 60 \\
\hline $\begin{array}{l}\text { Highest resolution } \\
(\Omega / \mathrm{mm})\end{array}$ & 5 & 1.6 & 0.5 \\
\hline
\end{tabular}

1

2

3

4 Tab. 1 Highest resolutions for different noise levels

5 